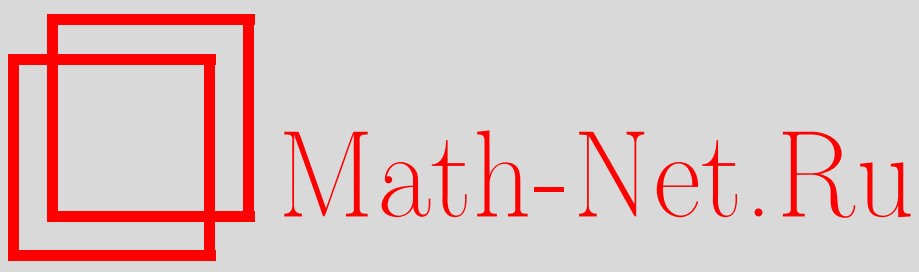

D. V. Lytkina, V. D. Mazurov, On the periodic groups saturated with finite simple groups of lie type $b_{3}$, Sibirsk. Mat. Zh., 2020, Volume 61, Number 3, 634-640

DOI: https://doi.org/10.33048/smzh.2020.61.311

Use of the all-Russian mathematical portal Math-Net.Ru implies that you have read and agreed to these terms of use http://www . mathnet.ru/eng/agreement

Download details:

IP: 54.197 .130 .99

April 26, 2023, 15:18:39

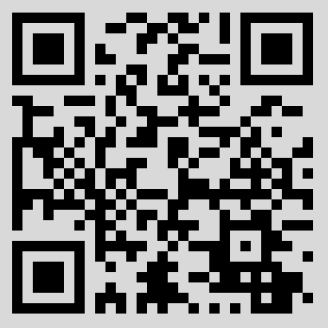


Сибирский математический журнал Май-июнь, 2020. Том 61, № 3

УДК 512.44

\title{
ПЕРИОДИЧЕСКИЕ ГРУППЫ, НАСЫЩЕННЫЕ КОНЕЧНЫМИ ПРОСТЫМИ ГРУППАМИ ЛИЕВА ТИПА $B_{3}$
}

\author{
Д. В. Лыткина, В. Д. Мазуров
}

\begin{abstract}
Аннотация. Пусть $\mathfrak{M}$ - некоторое множество конечных групп. Для группы $G$ через $\mathfrak{M}(G)$ обозначим множество всех подгрупп группы $G$, изоморфных элементам из $\mathfrak{M}$. Говорят, что группа $G$ насыщена группами из $\mathfrak{M}$ (для краткости, насыщена множеством $\mathfrak{M})$, если любая конечная подгруппа группы $G$ содержится в некотором элементе из $\mathfrak{M}(G)$. Доказывается, что периодическая группа $G$, насыщенная множеством $\mathfrak{M}=\left\{O_{7}(q) \mid q \equiv \pm 3(\bmod 8)\right\}$, изоморфна $O_{7}(F)$ для некоторого локально конечного поля $F$ нечетной характеристики.
\end{abstract}

DOI 10.33048/smzh.2020.61.311

Ключевые слова: периодическая группа, группа лиева типа, ортогональная группа, насыщенность.

\section{K 80-летию Юрия Леонидовича Ершова}

Пусть $\mathfrak{M}$ - некоторое множество групп. Говорят, что группа $G$ насыщена группами из $\mathfrak{M}$ (для краткости, насыщена множеством $\mathfrak{M}$ ), если все конечные подгруппы группы $G$ лежат в подгруппах $G$, изоморфных элементам из $\mathfrak{M}$.

Пусть $L-$ простая группа лиева типа [1] и $X$ - лиев тип группы $L$, а именно, $A_{n}, n \geq 1 ; B_{n}, n \geq 2 ; C_{n}, n \geq 3 ; D_{n}, n \geq 4 ; E_{6} ; E_{7} ; E_{8} ; F_{4} ; G_{2} ;$ ${ }^{2} A_{n}, n \geq 2 ;{ }^{2} B_{2} ;{ }^{2} D_{n}, n \geq 4 ;{ }^{3} D_{4} ;{ }^{2} E_{6} ;{ }^{2} F_{4}$ или ${ }^{2} G_{2}$. Из [2-6] следует, что локально конечная группа $G$, насыщенная конечными простыми группами типа $X$, изоморфна группе $X(F)$ типа $X$ над некоторым локально конечным полем $F$.

Известно, что условие локальной конечности группы можно заменить условием ее периодичности для типов $A_{1}, A_{2},{ }^{2} A_{2}, B_{2},{ }^{2} B_{2},{ }^{2} G_{2}$ [7-11]. Кроме того, периодическая группа, насыщенная одним из множеств $\left\{{ }^{3} D_{4}(q) \mid q\right.$ нечетно $\}$ или $\left\{G_{2}(q) \mid q\right.$ нечетно $\}$, изоморфна ${ }^{3} D_{4}(F)$ или соответственно $G_{2}(F)$ для некоторого локально конечного поля $F$ нечетной характеристики $[12,13]$.

Цель этой работы - получить аналогичный результат для множества, состоящего из групп $B_{3}(q)$, где $q \equiv \pm 3(\bmod 8)$.

Теорема. Пусть $G$ - периодическая группа, насыщенная множеством $\mathfrak{M}=\left\{B_{3}(q) \mid q \equiv \pm 3(\bmod 8)\right\}$. Тогда $G$ изоморфна группе $B_{3}(F)$ для некоторого локально конечного поля $F$ нечетной характеристики.

Мы используем стандартные обозначения и определения. Их можно найти, например, в [14].

Работа Д. В. Лыткиной выполнена при поддержке Математического центра в Академгородке (соглашение с Министерством науки и высшего образования Российской Федерации номер 075-15-2019-1613); работа В. Д. Мазурова выполнена за счет Российского научного фонда (проект № 19-11-00039).

(c) 2020 Лыткина Д. В., Мазуров В. Д. 
Периодические группы, насыщенные конечными простыми группами 635

Нам будет удобно воспринимать простые группы лиева типа $B_{3}$ над полями нечетной характеристики как ортогональные группы размерности 7 и использовать соответствующий геометрический язык.

Для описания строения конечных групп используем обозначения из [15]. Всю информацию об изучаемых группах, не упомянутую в тексте, можно найти в $[15,16]$.

\section{$\S 1$. Предварительные результаты}

Лемма 1.1. Пусть $q-$ степень простого числа и $q \equiv \varepsilon 5(\bmod 8)$, где $\varepsilon \in$ $\{+,-\}$. Обозначим через $L=L(q)$ простую группу $O_{7}(q)=\Omega_{7}(q)$ порядка $q^{9}\left(q^{6}-1\right)\left(q^{4}-1\right)\left(q^{2}-1\right)$.

(1) Силовская 2-подгруппа группы $L$ изоморфна силовской 2-подгруппе знакопеременной группы $\operatorname{Alt}(\Delta)$, где $\Delta=\{1,2, \ldots, 12\}$.

(2) Пусть

$$
\begin{gathered}
B_{1}=\langle(9,10)(11,12),(9,11)(10,12),(9,10)\rangle, \\
B_{2}=\langle(1,2)(3,4),(1,3)(2,4),(1,2),(1,5)(2,6)(3,7)(4,8)\rangle .
\end{gathered}
$$

Тогда $B_{1}-$ силовская 2-подгруппа симметрической группы $\operatorname{Sym}(\{9,10,11,12\})$, $B_{2}-$ силовская 2-подгруппа группы $\operatorname{Sym}(\{1,2, \ldots, 8\}), B=B_{1} \times B_{2}-$ силовская 2 -подгруппа группы $\operatorname{Sym}(\Delta)$ и $A=B \cap \operatorname{Alt}(\Delta)-$ силовская 2-подгруппа группы $\operatorname{Alt}(\Delta)$.

Кроме того, $A=C \lambda D$, где

$$
C=\langle(9,10)(11,12),(9,11)(10,12)\rangle,
$$

$D=\langle(1,2)(3,4),(1,3)(2,4),(1,5)(2,6)(3,7)(4,8),(1,2)(9,10)\rangle \simeq(2 \prec 2) \prec 2$.

Подгруппа

$$
T=\langle(5,6)(7,8),(5,7)(6,8),(9,10)(11,12), \$ 9,11)(10,12),
$$

$$
(1,2)(3,4),(1,3)(2,4)(5,7)(6,8)\rangle
$$

является элементарной абелевой подгруппой порядка 64 в $A$.

(3) Пусть

$$
a=(9,10)(11,12), \quad b=(1,2)(3,4)(5,6)(7,8), \quad c=a b .
$$

Тогда $\langle a, b\rangle$ - центр $A$. Количество элементов $x \in A$, для которых выполнено $x^{2}=a, x^{2}=b$ или $x^{2}=c$, равно $32,48,0$ соответственно (условимся впредь инволюцию $i \in A$ такую, что $A$ не содержит элемента $x$, для которого $x^{2}=i$, называть инволюиией нулевого типа).

Существует в точности две элементарные абелевы подгруппы $K_{1}$ и $K_{2}$ порядков 4 в $A$ такие, что $c \in K_{1} \cap K_{2}, K_{i}(i=1,2)$ содержит инволюцию нулевого типа, отличную от $c$, и $\left|C_{A}\left(K_{i}\right)\right|=256$. Кроме того, $K_{1}$ и $K_{2}$ сопряжены в $A$.

(4) Отождествим с $A$ из п. (2) одну из силовских 2-подгрупп группы $L$. Тогда $L$ содержит в точности три класса сопряженных инволюций. Их представителями являются $a, b, c$ и

$$
\begin{gathered}
C_{L}(a) \simeq\left(\Omega_{3}(q) \times \Omega_{4}^{+}(q)\right) \cdot 2^{2}=\left(\left(S L_{2}(q) * S L_{2}(q)\right) \times L_{2}(q)\right) \cdot 2^{2} ; \\
C_{L}(b) \simeq\left(\Omega_{2}^{\varepsilon}(q) \times \Omega_{5}(q)\right) \cdot 2^{2}=\Omega_{5}(q): D_{q-\varepsilon 1} \\
C_{L}(c) \simeq \Omega_{6}^{\varepsilon}(q) .2
\end{gathered}
$$


и $C_{L}(c)-$ максимальная подгруппа в $L$.

(5) Пусть $K_{1}, K_{2}$ - подгруппы, определенные в (3). Тогда $C_{L}\left(K_{i}\right)=K_{i} \times W_{i}$ при $i=1,2$, где $W_{i} \simeq \Omega_{5}(q)$ и $C_{L}\left(K_{i}\right)$ - максимальная подгруппа группы $C_{L}(c)$. Если $T$ - группа, определенная в $(2)$, то $C_{L}(T)=T$ и $N_{L}(T)=T: U$, где $U \simeq A_{7}$. Кроме того, $T \leq C_{A}\left(K_{1}\right) \cap C_{A}\left(K_{2}\right)$.

ДокАЗАТЕЛЬСтво. (1) доказано в [17], (2) и (3) доказываются прямой проверкой (вручную или с использованием, например, GAP [18]).

(4) Пусть $V-7$-мерное ортогональное пространство над полем порядка $q$, на котором действует $L$, и $t-$ инволюция из $A$. Поскольку $t^{2}=1$ и $\operatorname{det} t=1$, максимальная размерность $d_{t}$ подпространства $U_{t}$ в $V$, на котором $t$ действует тривиально, равна 1,3 или 5 и $V$ - ортогональная прямая сумма $V=U_{t} \oplus W_{t}$, где $t$ действует на $W_{t}$ как скаляр -1 . Следовательно, $C_{G O(V)}(t)=G O\left(U_{t}\right) \times$ $G O\left(W_{t}\right)$ и $C_{L}(t)$ содержит $\Omega\left(U_{t}\right) \times \Omega\left(W_{t}\right)$.

Сначала предположим, что $d_{t}=1$. Табл. 8.39 в [16] показывает, что $C_{L}(t)$ максимальна в $L$ и изоморфна $\Omega_{6}^{+}(q) .2$ или $\Omega_{6}^{-}(q) .2$. Поскольку для $\varepsilon=+$ центр группы $\Omega_{6}^{-}(q) .2$ тривиален, в этом случае $C_{L}(t) \simeq \Omega_{6}^{+}(q) .2$. Точно так же $C_{L}(t) \simeq \Omega_{6}^{-}(q) .2$, если $\varepsilon=-$.

Аналогично если $d_{t}=5$, то

$$
C_{L}(t) \simeq\left(\Omega_{2}^{\varepsilon}(q) \times \Omega_{5}(q)\right) .2^{2} \simeq\left((q-\varepsilon 1) / 2 \times \Omega_{5}(q)\right) .2^{2} \simeq \Omega_{5}(q) \lambda D_{2(q-\varepsilon 1)} .
$$

Если $d_{t}=3$, то

$$
C_{L}(t) \simeq\left(\Omega_{3}(q) \times \Omega_{4}^{+}(q)\right) \cdot 2^{2} \simeq\left(L_{2}(q) \times\left(S L_{2}(q) * S L_{2}(q)\right)\right) .2^{2} .
$$

В частности, по лемме Витта $L$ содержит в точности три класса сопряженных инволюций и сравнение $\left|C_{L}(t)\right|$ с $|L|$ показывает, что все инволюции из $L$ лежат в центре некоторой силовской 2-подгруппы группы $L$, т. е. сопряжены с $a, b$ или $c$.

В случае, когда $d_{t} \neq 1, C_{L}(t)$ содержит такой элемент $x \in A$, что $x^{2}=t$, поэтому в силу (3) если $d_{t}=1$, то $t$ сопряжена с $c$ в $L$. Более того, если $d_{t}=5$, то число элементов $x$, для которых $x^{2}=t$ в $A \cap\left(S L_{2}(q) * S L_{2}(q)\right) \times L_{2}(q)$, больше 32 . Значит, в силу (3) $t$ сопряжена с $b$.

(5) Сохраним обозначения из (4), а также его доказательства. Пусть $u-$ инволюция в $C_{A}(c)$, которая сопряжена с $c$ в $L$ и действует как -1 на $U_{c}$. Пусть $K=\langle c, u\rangle$. Тогда $W_{c}=W_{c}^{-} \oplus W_{c}^{+}$, где $w u=w$ для $w \in W_{c}^{+}, w u=-w$ для $w \in W_{c}^{-}$и $\operatorname{dim}\left(W_{c}^{+}\right)=1, \operatorname{dim}\left(W_{c}^{-}\right)=5$. Более того, действие $C_{L}(K)$ на $W_{c}^{-}$содержит $\Omega\left(W_{c}^{-}\right)$и, следовательно, $C_{L}(K)$ содержит подгруппу $K \times$ $\Omega\left(W_{c}^{-}\right)$, изоморфную $2^{2} \times \Omega_{5}(q)$. Далее, информация из [16, табл. 8.31, 8.33, 8.39] показывает, что $C_{L}(K)$ - максимальная подгруппа в $C_{L}(c) \simeq \Omega_{6}^{\varepsilon}(q) .2$ и $C_{L}(K) \simeq 2^{2} \times \Omega_{5}(q)$. Имеем $\left|A: C_{A}(K)\right|=2$, поэтому $K=K_{1}$ или $K=K_{2}$ в силу (3), а поскольку $\Omega_{5}(q)$ содержит элементарную абелеву подгруппу порядка $16, C_{A}(K)$ содержит элементарную абелеву подгруппу порядка 64 и в силу (3) эта подгруппа совпадает с $T$. Остальные утверждения п. (5) следуют из $[16$, табл. 8.39].

\section{§ 2. Доказательство теоремы}

Пусть $G$ - периодическая группа, насыщенная множеством

$$
\mathfrak{M}=\left\{\Omega_{7}(q) \mid q \equiv \varepsilon 5(\bmod 8), \varepsilon \in\{+,-\}\right\},
$$

и $\mathfrak{M}(G)$ - множество подгрупп группы $G$, изоморфных элементам из $\mathfrak{M}$. Зафиксируем $L \in \mathfrak{M}(G)$ и отождествим ее с $L=\Omega_{7}(q)$ из п. (1); сохраним также все введенные там обозначения. Для ясности пусть $K=K_{1}$. Положим $H=C_{G}(K)$. 
Лемма 2.1. Все силовские 2-подгруппы группы $G$ сопряжены с $A$. Если $x, y$ лежат в центре $A$ и сопряжены в $G$, то $x=y$.

ДоказАтеЛьство. Пусть $x=y^{g}$, где $g \in G$. Тогда $x \in Z\left(A^{g}\right)$ и $A^{g} \leq$ $C_{G}(x)$. По лемме Шункова [19, предложение 8] $A$ сопряжена с $A^{g}$ в $C_{G}(x)$, т. е. $A=A^{g u}$, где $u \in C_{G}(x)$. По лемме $1.1(3) x^{g u}=x$ и $y=x^{g^{-1}}=x^{u^{-1} g^{-1}}=x$.

Лемма 2.2. Пусть $\bar{H}=H / K$. Тогда $H \simeq \Omega_{5}(F)$ для некоторого локально конечного поля $F$ нечетной характеристики и, в частности, $H$ локально конечна. Более того, $Н$ является объединением возрастающей цепочки

$$
H_{1} \leq H_{2} \leq \cdots \leq H_{i} \leq \cdots
$$

подгрупп $H_{i}=K \times M_{i}$, где $M_{i} \simeq \Omega_{5}\left(q_{i}\right), q_{i} \equiv \varepsilon 5(\bmod 8)$.

ДокАЗАтельство. Пусть $\bar{R}-$ конечная подгруппа в $\bar{H}$ и $R-$ ее полный прообраз в $H$. Тогда $R$ конечна и, следовательно, содержится в $C_{L^{*}}(K) \leq$ $C_{L^{*}}(c)$, где $L^{*}$ - подгруппа $G$, изоморфная $\Omega_{7}\left(q^{*}\right)$ для некоторого конечного $q^{*}$.

Так как $c \in K$, по лемме 1.1(3) существует такая силовская 2-подгруппа $A^{*}$ группы $L^{*}$, что $c \in Z\left(A^{*}\right)$, и, следовательно, $A^{*}$ сопряжена с $A$ в $L^{*}$, поскольку $A$ - силовская 2-подгруппа группы $G$. По лемме $2.1 A^{x}=A^{*}$ для некоторого $x \in G$ и $c^{x} \in Z\left(A^{*}\right)$. Снова по лемме 2.1 с $A^{*}$ вместо $A$ имеем $c^{x}=c$. Таким образом, $c$ - инволюция нулевого типа в $A^{*}$ и $K=K_{1}^{*}$ или $K_{2}^{*}$, где $K_{i}^{*}$ удовлетворяет условиям для $K_{i}, i=1,2$, после замены $A$ на $A^{*}$ (см. лемму 1.1(3)). Следовательно, $C_{L^{*}}(K)=K \times M$, где $M \simeq \Omega_{5}\left(q^{*}\right)$ и $\bar{R}$ лежит в подгруппе группы $\bar{H}$, изоморфной $\Omega_{5}\left(q^{*}\right)$. Это доказывает, что $\bar{H}$ насыщена группами, изоморфными $\Omega_{5}(q)$. Поскольку $\Omega_{5}(q) \simeq S_{4}(q)$, то $\bar{H}$ изоморфна $\Omega_{5}(F)$ в силу [12]. Отсюда следует заключение леммы.

Лемма 2.3. Пусть $T$ - подгруппа $C_{L}(K)$, определенная в лемме 1.1(5). Тогда

$$
C_{H}(T)=C_{G}(T)=T, \quad N=N_{G}(T)=T U,
$$

где $U \simeq \operatorname{Alt}(7)$. Кроме того, $N \cap H=K \times N_{0}$, где $N_{0} \simeq 2^{4}: \operatorname{Alt}(5) ; N \cap C_{G}(c)=$ $\langle c\rangle \times N_{1}$, где $N_{1} \simeq 2^{5}: \operatorname{Alt}(6)$. Более того, $N, N_{0}, N_{1}$ действуют неприводимо сопряжением в $G$ на $\mathrm{O}_{2}(\mathrm{~N}), \mathrm{O}_{2}\left(N_{0}\right), \mathrm{O}_{2}\left(N_{1}\right)$ соответственно.

ДоказАтельство. Пусть $r \in C_{G}(T)$. Тогда $\langle T, r\rangle$ - конечная группа, которая по условию содержится в подгруппе $R \simeq \Omega_{7}\left(q^{*}\right)$ для некоторого $q^{*}$. По лемме 1.1(5) с $q^{*}$ вместо $q$ имеем $C_{R}(T)=T$, откуда $C_{G}(T)=T$. Следовательно, $N_{G}(T)$ конечна, а потому лежит в подгруппе группы $G$, изоморфной $\Omega_{7}\left(q^{*}\right)$ для некоторого $q^{*}$. В силу леммы $1.1(5) N_{G}(T)=T U$, где $U \simeq \operatorname{Alt}(7)$. Остальные утверждения леммы следуют из информации о подгруппах группы $\Omega_{7}(q)$ в [16].

Лемма 2.4. $H$ содержится в подгруппе $P$ из $C_{G}(c)$, которая является объединением возрастающей цепочки подгрупп $P_{i}, i \in \mathbb{N}$, изоморфных $\Omega_{6}^{\varepsilon}\left(q_{i}\right) .2$, $q_{i} \equiv \varepsilon 5(\bmod 8)$.

ДоКАЗАТЕЛЬСтво. По лемме $2.3 H$ является объединением возрастающей последовательности

$$
H_{1}<H_{2}<\cdots<H_{n}<\ldots,
$$

где

$$
H_{i}=K \times M_{i}, \quad M_{i} \simeq \Omega_{5}\left(q_{i}\right), i=1,2, \ldots, \quad H_{1}=C_{L}(K) \geq C_{A}(K) \geq T .
$$


По условию $H_{i} \leq R_{i} \simeq \Omega_{7}\left(q_{i}^{*}\right)$ для некоторого $q_{i}^{*}$. Если $P_{i}=C_{R_{i}}(c)$, то $P_{i} \simeq \Omega_{6}^{\varepsilon}\left(q_{i}^{*}\right) .2$ и $H_{i}-$ максимальная подгруппа в $P_{i}$. Поскольку $P_{i} \geq N_{1} \not H_{i}$, то

$$
P_{i}=\left\langle H_{i}, N_{1}\right\rangle \leq\left\langle H_{i+1}, N_{1}\right\rangle
$$

и $P_{1} \leq P_{2} \leq \ldots$ образуют возрастающую последовательность. Ее объединение - это искомая подгруппа $P$.

Лемма 2.5. $P=C$.

ДокАЗАтЕЛьство. Предположим противное и рассмотрим $t \in C_{G}(c) \backslash P$. Тогда $\left\langle K, K^{t}\right\rangle$ конечна, а значит, содержится в подгруппе $D$, лежащей в $C_{G}(c)$ и изоморфной $\Omega_{6}^{\varepsilon}(q) .2$ для некоторого $q$. Пусть $\Gamma-$ граф с множеством вершин $\Sigma=\left\{K^{d} \mid d \in D\right\}$, где две различные вершины $K^{r}$ и $K^{s}$ соединены ребром тогда и только тогда, когда $\left[K^{r}, K^{s}\right]=1$. Пусть $\Delta-$ компонента связности графа $\Gamma$, содержащая $K$. Поскольку $K \leq T$ и $N_{1} \neq N_{0}$ (см. лемму 2.3), $K^{N_{1}} \neq\{K\}$, а следовательно, $|\Delta| \geq 2$. Таким образом, если $\Delta \neq \Sigma$, то $D$ действует на $\Sigma$ сопряжением транзитивно, но импримитивно, а значит, стабилизатор вершины $K$ в $D$, который равен $N_{D}(K)$, не максимален в $D$, что противоречит лемме $1.1(5)$. Значит, $\Delta=\Sigma$ и существует такая последовательность $t_{1}, t_{2}, \ldots, t_{m}=t$ элементов из $C_{G}(c)$, что

$$
1=\left[K, K^{t_{1}}\right]=\left[K^{t_{i}}, K^{t_{i+1}}\right], \quad i=1,2, \ldots, m-1 .
$$

Докажем индукцией по $m$, что $K^{t_{m}} \leq P$. Если $m=1$, то $K^{t_{1}} \leq C_{G}(K)$, и по лемме $2.3 K^{t_{1}} \leq P$. Следовательно, для некоторого $u \in P$ выполнено $K^{t_{1} u}=K$ и

$$
1=\left[K, K^{t_{2} u}\right]=\left[K^{t_{i} u}, K^{t_{i+1} u}\right], \quad i=2, \ldots, m .
$$

По предположению индукции $K^{t_{m} u} \leq P$ и $K^{t_{m}} \leq P$.

Итак, для любого $t \in C_{G}(c)$ выполнено $K^{t} \leq P$.

Отсюда следует, что $P^{t}=C_{G}(c) \cap C_{G}\left(K^{t}\right) \leq P$, а значит, $P \unlhd C_{G}(c)$. Поскольку $K^{t}$ и $K$ сопряжены в $P$, то

$$
C_{G}(c)=P N_{G}(K)=P\left(C_{G}(K) \cdot N_{P}(K)\right) \leq P,
$$

и лемма доказана.

Лемма 2.6. $C_{G}(c)$ лежит в $M \leq G$, изоморфной $\Omega_{7}(F)$ для некоторого локально конечного поля $F$.

ДокАЗАтЕЛьство. По лемме $2.5 C_{G}(c)$ является объединением возрастающей цепочки

$$
P_{1} \leq P_{2} \leq \cdots \leq P_{i} \leq \ldots, \quad i \in \mathbb{N}
$$

подгрупп из $G$ таких, что каждая из $P_{i}$ изоморфна $\Omega_{6}^{\varepsilon}\left(q_{i}\right) .2$ для некоторого $q_{i} \equiv$ $\varepsilon 5(\bmod 8)$. Поскольку $P_{i}$ конечна, она содержится в некоторой подгруппе $L_{i}^{*} \leq$ $G$, изоморфной $\Omega_{7}\left(q_{i}^{*}\right)$ для некоторого $q_{i}^{*}$. В силу леммы $2.3 L_{i}^{*}$ содержит $N_{G}(T)$, а значит, $G$ содержит возрастающую последовательность конечных подгрупп

$$
L_{i}=\left\langle P_{i}, N_{G}(T)\right\rangle, \quad i \in \mathbb{N} .
$$

Докажем, что $L_{i} \simeq \Omega_{7}\left(q_{i}\right)$. В самом деле, по предположению $L_{i}$ лежит в подгруппе $L_{i}^{*} \simeq \Omega_{7}\left(q^{*}\right)$ и $q_{i}$ делит $q^{*}$.

Очевидно, $A \leq L_{i}^{*}$, откуда $C_{L_{i}^{*}}(c) \simeq \Omega_{6}^{\varepsilon}\left(q^{*}\right) .2$. Следовательно, $C_{L_{i}^{*}}(c) \geq P_{i}$, и $L_{i}^{*}$ содержит подгруппу $M_{i}$, изоморфную $\Omega_{7}\left(q_{i}\right)$, которая содержит $P_{i}$ как подгруппу группы $C_{L_{i}}(c)$. Таким образом,

$$
M_{i}=\left\langle P_{i}, N_{L_{i}^{*}}(T)\right\rangle=\left\langle P_{i}, N_{G}(T)\right\rangle=L_{i}
$$


Периодические группы, насыщенные конечными простыми группами 639

откуда $L_{i} \simeq \Omega_{7}(q)$ и $C_{L_{i}}(c)=P_{i}$. В частности, $P_{i}-$ максимальная подгруппа группы $L_{i}$ по лемме $1.1(3)$.

Пусть $M$ - объединение последовательности (1). По любому из результатов из [3-6] $M \simeq \Omega_{7}(F)$, где $F-$ локально конечное поле нечетной характеристики.

Лемма 2.7. $M=G$.

ДоказАтельство. Пусть $g \in G$ и $t=c^{g}$. Тогда $\left\langle c, c^{g}\right\rangle$ конечна и, следовательно, содержится в подгруппе $R$ группы $G$, изоморфной $\Omega_{7}(r)$ для некоторого $r$.

Пусть $\Delta-$ множество инволюций, принадлежащих $R$ и сопряженных с $c$ в $G$. Определим граф $\Gamma$ с множеством вершин $\Delta$, где вершины $c^{g_{1}}$ и $c^{g_{2}}$ соединены ребром тогда и только тогда, когда $\left[c^{g_{1}}, c^{g_{2}}\right]=1$. Поскольку $C_{R}(c)-$ максимальная подгруппа группы $R$ и $\left|C_{R}(c) \cap \Delta\right| \geq 2$, заключаем, как и в доказательстве леммы 2.5, что граф $Г$ связен. Отсюда вытекает, что $c^{G} \subseteq M$, $\left\langle c^{G}\right\rangle=M$, откуда $M \unlhd G$.

Пусть $g \in G$. Поскольку $M$ локально конечна, $\langle c, g\rangle$ конечна и, значит, содержится в подгруппе $R$ группы $G$, изоморфной $\Omega_{7}(r)$ для некоторого $r$. Так как $\left\langle c^{R}\right\rangle=R$, то $g$ лежит в $\left\langle c^{R}\right\rangle \leq M$.

Лемма доказана. Она завершает доказательство теоремы.

\section{ЛИТЕРАТУРА}

1. Carter R. W. Simple groups of Lie type. London: John Wiley \& Sons, 1972.

2. Беляев В. В. Локально конечные группы Шевалле // Исследования по теории групп. Свердловск: УНЦ АН СССР, 1984. С. 39-50.

3. Боровик A. В. Вложения конечных групп Шевалле и периодические линейные группы // Сиб. мат. журн. 1983. Т. 24, № 6. С. 26-35.

4. Hartley B., Shute G. Monomorphisms and direct limits of finite groups of Lie type // Q. J. Math. Ser. 2. 1984. V. 35, N 137. P. 49-71.

5. Thomas $S$. The classification of the simple periodic linear groups // Arch. Math. 1983. V. 41. P. 103-116.

6. Larsen M. J., Pink R. Finite subgroups of algebraic groups // J. Amer. Math. Soc. 2011. V. 24, N 4. P. 1105-1158.

7. Шлепкин A. K. О некоторых периодических группах, насыщенных конечными простыми группами // Мат. тр. 1998. Т. 1, № 1. С. 129-138.

8. Рубашкин А. Г., Филиппов К. А. О периодических группах, насыщенных группами $L_{2}\left(p^{n}\right) / /$ Сиб. мат. журн. 2005. Т. 46, № 6. С. 1388-1392.

9. Лыткина Д. В., Шлепкин А. А. Периодические группы, насыщенные конечными простыми группами типов $U_{3}$ и $L_{3} / /$ Алгебра и логика. 2016. Т. 55, № 4. С. 441-448.

10. Филиппов K. А. Группы, насыщенные конечными неабелевыми простыми группами и их расширениями: Дис. ... канд. физ.-мат. наук. Красноярск, 2005.

11. Филиппов K. А. О периодических группах, насыщенных конечными простыми группами // Сиб. мат. журн. 2012. Т. 53, № 2. С. 430-438.

12. Лыткина Д. В., Мазуров В. Д. Характеризация простых симплектических групп размерности 4 над локально конечными полями в классе периодических групп // Алгебра и логика. 2018. Т. 57, № 3. С. 306-320.

13. Вей С., Го В., Лыткина Д. В., Мазуров В. Д. Характеризация локально конечных простых групп типа ${ }^{3} D_{4}$ над полями нечетных характеристик в классе периодических групп // Сиб. мат. журн. 2018. Т. 59, № 5. С. 1013-1019.

14. Isaacs I. M. Finite group theory. Providence, RI: Amer. Math. Soc., 2008. (Grad. Stud. Math.; V. 92).

15. Conway J. H., Curtis R. T., Norton S. P., Parker R. A., Wilson R. A. Atlas of finite groups. Oxford: Clarendon Press, 1985.

16. Bray J. N., Holt D. F., Roney-Dougal C. M. The maximal subgroups of the low-dimensional finite classical groups. Cambridge: Camb. Univ. Press, 2013. (Lond. Math. Soc. Lect. Note Ser.). 
17. Wong W. J. Twisted wreath products and Sylow 2-subgroups of classical simple groups // Math. Z. 1967. Bd 97, Heft 5. S. 406-424.

18. GAP: Groups, algorithms, and programming. http://www/gap-system.org.

19. Лыткина Д. В., Тухватуллина Л. Р., Филиппов К. А. Периодические группы, насыщенные конечными простыми группами $U_{3}\left(2^{m}\right) / /$ Алгебра и логика. 2008. Т. 47, № 3. C. $288-306$.

Поступила в редакиию 17 января 2020 г.

После доработки 17 января 2020 г.

Принята к публикации 19 февраля 2020 г.

Лыткина Дарья Викторовна

Сибирский государственный университет телекоммуникаций и информатики,

ул. Кирова, 86, Новосибирск 630102;

Новосибирский государственный университет,

ул. Пирогова, 1, Новосибирск 630090

daria.lytkin@gmail.com

Мазуров Виктор Данилович

Институт математики им. С. Л. Соболева СО РАН,

пр. Академика Коптюга, 4, Новосибирск 630090

mazurov@math.nsc.ru 